\title{
Molecular Type Permutation Shift Keying for Molecular Communication
}

\author{
Yuankun Tang, Miaowen Wen, Senior Member, IEEE, Xuan Chen, Yu Huang, and Lie-Liang Yang, Fellow, IEEE
}

\begin{abstract}
Molecular communication (MC) via diffusion is envisioned to be a new paradigm for information exchange in the future nanonetworks. However, the strong inter-symbol interference (ISI) caused by the diffusion channel significantly deteriorates the performance of MC systems. To this end, we propose a novel modulation technique to reduce the ISI effect, termed as molecular type permutation shift keying (MTPSK), which encodes information on the permutations of multiple types of molecules. We design a Genie-aided maximum-likelihood detector and a conventional maximum-likelihood detector, and analyze their performance in terms of bit error rate (BER). Aiming at lower computational complexity, we further design a low-complexity maximum-likelihood detector using a Viterbi-like algorithm with compromised error performance. BER simulation results corroborate that the proposed MTPSK can outperform the prevailing modulation schemes for MC, including molecular shift keying (MoSK), concentration shift keying, depleted MoSK, and pulse position modulation.
\end{abstract}

Index Terms-Inter-symbol interference, modulation, molecular communication, maximum-likelihood, Viterbi.

\section{INTRODUCTION}

$\mathbf{M}$ OLECULAR communication (MC) is an emerging communication paradigm that conveys information via chemical signals. Inspired by nature, numerous MC systems were proposed in [1]. Among them, molecular communication via diffusion $(\mathrm{MCvD})$ is an effective and energy-efficient method, where the emitted molecules propagate through the environment based on Brownian motion. However, due to the insufficient dynamics of diffusion channel, the channel impulse response has a long tail that may last several symbol durations in the MCvD system, especially when the data rate is high. As shown in [2] and [3], the resultant inter-symbol interference (ISI) poses a great challenge to MCvD in terms of reliability.

In the literature, various modulation schemes were proposed, including the concentration shift keying (CSK) [4], molecular shift keying (MoSK) [4], pulse position modulation (PPM) [5], and space shift keying-based MC (SSK-MC) [6], [7], which encode messages on the concentration, type, release time, and spatial index of transmitters, respectively. In MoSK, multiple types of molecules are used for encoding.

Y. Tang, M. Wen, X. Chen and Y. Huang are with the School of Electronics and Information Engineering, South China University of Technology, Guangzhou, China (e-mail: \{eeyktang, eechenxuan, ee06yuhuang\}@mail.scut.edu.cn; eemwwen@scut.edu.cn).

L.-L. Yang is with the Southampton Wireless Group, School of Electronics and Computer Science, University of Southampton, Southampton SO17 1BJ, U.K. (e-mail: 1ly@ecs.soton.ac.uk).

This work was supported in part by the Natural Science Foundation of Guangdong Province under Grant 2018B030306005, in part by the National Natural Science Foundation of China under Grant 61871190, and in part by the Fundamental Research Funds for the Central Universities under Grant 2019SJ02. (Corresponding author: Miaowen Wen.)
Since the transmission interval of the same type of molecules is statistically enlarged, MoSK is less susceptible to the ISI effect than the CSK technique that depends on only one type of molecules. Combining CSK with MoSK, depleted MoSK (D-MoSK), was proposed in [8], where multiple independent CSK streams are simultaneously transmitted over orthogonal channels. D-MoSK significantly increases the transmitted bits per symbol. Therefore, it reduces the effect of ISI in comparison with CSK and MoSK at the same data rate. For PPM, since its decoder aims to identify the intended position of pulse by choosing the position having the maximum number of molecules among all candidates, the effect of ISI declines [5]. However, the optimal number of candidate positions per symbol, dependent on the system parameters, affects the detection performance significantly. Another ISI-mitigation modulation technique, named Molecular ARray-based COmmunication (MARCO), was presented in [9]. It encodes information on the releasing order of molecules of two types, i.e., bit- 0 was encoded by releasing one type I molecule followed by one type II molecule, and bit-1 was encoded by releasing one type II molecule followed by one type I molecule.

In this paper, we propose a novel modulation technique, termed as molecular type permutation shift keying (MTPSK), which encodes information on the permutations of multiple types of molecules. The number of molecular types in MTPSK can be an arbitrary integer larger than one, thus subsuming MARCO as a special case. Unlike MARCO that transmits a single molecule each time to a 1-D channel, MTPSK emits pulses of molecules to a 3-D channel. Our studies show that MTPSK has in particular the advantage for MC to enhance its immunity to the strong ISI, and hence the bit error rate (BER) performance. We propose a Genieaided maximum-likelihood (GML) detector and a conventional maximum-likelihood (CML) detector for MTPSK to attain its ultimate performance of ISI mitigation. Upper bounds on the resulting BERs are derived. For practical implementation, a low-complexity maximum-likelihood (LML) detector using a Viterbi-like algorithm is also proposed at the cost of a slight performance loss. Finally, we compare MTPSK with CSK, MoSK, D-MoSK, and PPM, in order to validate its superior BER performance in some operational conditions.

The rest of this paper is organized as follows. The system model and principle of MTPSK are presented in Section II. Section III designs the GML, CML, and LML detectors. BER upper bounds are derived in Section IV. Numerical simulation results in terms of BER are presented in Section V. Finally, Section VI concludes this paper. 


\section{System MOdEL AND MTPSK PRINCIPLE}

The proposed MTPSK scheme may be operated with various channel models. However, for demonstration purpose we consider an MCvD system in a 3-D unbounded environment with a point transmitter source and a passive spherical receiver. The transmitter stores molecules of $M$ types and emits pulses of molecules at a time. The emitted molecules propagate through the fluid medium via diffusion without any chemical reactions. All of the molecules are assumed to be the isomers sharing the same diffusion coefficient $D[10]$. The passive receiver can distinguish these isomers of $M$ types and detect their concentrations correspondingly. Within its detection region, molecules are assumed to be homogeneously distributed, which is valid when the distance $d$ between the transmitter and receiver is sufficiently large in comparison to the radius $r$ of the receiver and the symbol duration $T_{s}$ is larger than or close to $d^{2} / 6 D$ [11].

To implement MTPSK, there are $M$ emission instants with an equal time interval $T_{e}$ during a $T_{s}$, namely $T_{s}=M T_{e}$. The transmitter emits only one out of $M$ types at each emission instant. The types of emitted molecules at $M$ emission instants during the same symbol duration are different from each other, and the information bits are encoded on their permutation. Since there are in total $M$ ! permutation candidates, $b=\left\lfloor\log _{2} M !\right\rfloor$ information bits can be transmitted per symbol potentially.

We choose $N=2^{b}$ out of the $M$ ! permutations as activation patterns, which form a symbol set $\mathbf{S}=\left\{\mathbf{S}_{1}, \mathbf{S}_{2}, \ldots, \mathbf{S}_{N}\right\}$. The $n$-th activation pattern $\mathbf{S}_{n}$ is an $M \times M$ permutation matrix with only one entry equal to one in each row and column, and all the others equal to zero, where $n \in\{1, \ldots, N\}$. The $(m, v+1)$-th entry of $\mathbf{S}_{n}$, denoted by $s_{n}(m, v+1)$, equaling one indicates that the transmitter emits $E_{e}$ molecules of the $m$-th type at time $t_{0}=v T_{e}$, where $m=1, \ldots, M$ and $v=0, \ldots, M-1$. According to the Fick's second law of diffusion [12], the expected molecular concentration of the $m$-th type at the receiver given the $n$-th activation pattern at time $t \in\left[v T_{e},(v+1) T_{e}\right]$ can be expressed as

$$
C_{m}(d, t)=\sum_{j=0}^{v} E_{e} p\left(d, t-j T_{e}\right) s_{n}(m, j),
$$

where

$$
p(d, t)=\frac{1}{(4 \pi D t)^{\frac{3}{2}}} \exp \left(-\frac{d^{2}}{4 D t}\right) .
$$

From (1), it is clear that the expected received concentration is corrupted by the previously emitted molecules from the same symbol, which is called inter-emission interference (IEI).

Consider that the transmitter emits a sequence of symbols in the form of activation patterns as $\mathbf{X}_{0}, \ldots, \mathbf{X}_{u}, \ldots$, where $u$ is an integer and $\mathbf{X}_{u} \in \mathbf{S}$. Denote $x_{w}(m)=x_{u}(m, v)$, where $w=u M+v$ and $x_{u}(m, v)$ is the $(m, v+1)$-th entry of $\mathbf{X}_{u}$. The corrupted molecular concentration of the $m$-th type at the receiver at time $t \in\left[w T_{e},(w+1) T_{e}\right]$ is given by

$$
y_{m}(d, t)=\sum_{j=0}^{w}\left[E_{e} p\left(d, t-j T_{e}\right)+n_{j}(t)\right] x_{j}(m),
$$

where $n_{j}(t)$ represents the counting noise induced by the $j$-th emitted molecules.

\section{RECEIVER DESIGN FOR MTPSK}

In this section, we derive the statistics of the received signal, based on which the GML and CML detectors are designed to show the potential of the MTPSK scheme. Furthermore, for practical implementation, we propose the LML detector using a Viterbi-like algorithm.

Assume that the receiver synchronizes with the transmitter and samples molecular concentrations after a time interval $t_{m}=d^{2} / 6 D$ from the emission instant for observing the maximum molecule concentration [13]. Therefore, the molecular concentration of the $m$-th type at the receiver at time $t=w T_{e}+t_{m}$ is given by

$$
y_{m}(w)=\sum_{j=0}^{w}\left[E_{e} p_{w-j}+n_{j}(w)\right] x_{j}(m),
$$

where $p_{w-j}$ and $n_{j}(w)$ denote $p\left(d,(w-j) T_{e}+t_{m}\right)$ and $n_{j}\left(w T_{e}+t_{m}\right)$, respectively.

Since the interference of those molecules sent long before the current symbol can be ignored, we restrict the ISI length to $L$. Consider that the MC system is at its stable state, i.e., $w \geq L M$, implying a sufficiently long ISI length for the current symbol detection. Therefore, based on (4), the received concentration can be expressed as

$$
\begin{aligned}
y_{m}(w)= & \sum_{j=u M}^{w}\left[E_{e} p_{w-j}+n_{j}(w)\right] x_{j}(m) \\
& +\sum_{j=(u-L) M}^{u M-1}\left[E_{e} p_{w-j}+n_{j}(w)\right] x_{j}(m) .
\end{aligned}
$$

From (5), it is observed that the desired concentration $x_{w}(m)$ experiences ISI from the previous symbols, given by the second term. Moreover, when $v \geq 1$, the IEI from the first term, corresponding to $j=u M, \ldots, w-1$, also interferes the desired concentration $x_{w}(m)$.

Denote $\lambda_{j}=E_{e} p_{w-j} V_{r}$ and $Z_{w}(m)=y_{m}(w) V_{r}$, where $V_{r}=\frac{4}{3} \pi r^{3}$ is the volume of the receiver and $Z_{w}(m)$ represents the number of molecules of the $m$-th type observed by the receiver at the $w$-th instant. Based on [12], $Z_{w}(m)$ follows a Poisson distribution, whose parameter is

$$
\begin{aligned}
\Lambda_{m}(w) & =\lambda_{w} x_{w}(m)+\sum_{j=u M}^{w-1} \lambda_{j} x_{j}(m)+\sum_{j=(u-L) M}^{u M-1} \lambda_{j} x_{j}(m) \\
& =\lambda_{w} x_{w}(m)+\Lambda_{I E I}+\Lambda_{I S I},
\end{aligned}
$$

where $\Lambda_{I E I}$ and $\Lambda_{I S I}$ denote the Poisson parameters of IEI and ISI, respectively. Recalling that $w=u M+v$, (6) can be alternatively rewritten as

$$
\Lambda_{m}^{v}(u)=\lambda_{u}^{v} x_{u}(m, v)+\Lambda_{I E I}+\Lambda_{I S I} .
$$

The probability mass function of $Z_{u}(m, v)$ is given by

$$
\operatorname{Pr}\left(Z_{m}^{v}=z_{m}^{v}\right)=\frac{1}{z_{m}^{v} !}\left[\Lambda_{m}^{v}(u)\right]^{z_{m}^{v}} e^{-\Lambda_{m}^{v}(u)},
$$

where we omit the notation $u$ in $Z_{m}^{v}$ and $z_{m}^{v}$ for brevity.

\section{A. GML Detector}

Based on (7), we design a GML detector which assumes the ideal knowledge of the previous $L$ symbols, i.e., $\Lambda_{I S I}$. Within the $u$-th symbol duration, the receiver observes the molecular numbers and stores them in memory until the $(M-1)$-th 
observation arrives. When $T_{e}>r^{2} / D$, these observations in terms of the molecular numbers of $M$ types are mutually independent according to [14]. Based on (8), a GML detection method can be expressed as

$$
\begin{aligned}
\hat{\mathbf{X}}_{u} & =\arg \max _{\widetilde{\mathbf{x}}_{u} \in \mathbf{S}} \sum_{m=1}^{M} \sum_{v=0}^{M-1} \ln \left(\operatorname{Pr}\left(Z_{m}^{v}=z_{m}^{v} \mid \widetilde{x}_{u}(m, v)\right)\right) \\
& =\arg \max _{\widetilde{\mathbf{x}}_{u} \in \mathbf{S}} \sum_{m=1}^{M} \sum_{v=0}^{M-1} z_{m}^{v} \ln \left(\Lambda_{m}^{v}\left(u \mid \widetilde{x}_{u}(m, v)\right)\right) \\
& =\arg \max _{\widetilde{\mathbf{x}}_{u} \in \mathbf{S}} \sum_{m=1}^{M} \sum_{v=0}^{M-1} z_{m}^{v} \ln \left(u \mid \widetilde{x}_{u}(m, v)\right)-\ln \left(z_{m}^{v} !\right)
\end{aligned}
$$

where the last equation holds since based on (7) we have

$$
\sum_{m=1}^{M} \sum_{v=0}^{M-1} \Lambda_{m}^{v}\left(u \mid \widetilde{x}_{u}(m, v)\right)=\sum_{v=0}^{M-1}(M-v) \lambda_{u}^{v}+\Lambda_{I S I},
$$

which is equal for all activation patterns.

Based on (9), the GML detector first calculates the simplified likelihood function and then selects the largest one among the $N$ activation patterns as the output symbol.

\section{B. CML Detector}

Although the GML detector exhibits the potential of MTPSK, it is difficult to obtain the exact $\Lambda_{I S I}$ in practice. Therefore, we propose a CML detector, which has no knowledge of the exact ISI but exploits the expectation of ISI, denoted by $\Lambda_{c}$, for detection. Given $\mathbf{X}_{u}, \Lambda_{c}$ can be expressed as

$$
\Lambda_{c}=E\left[\sum_{j=(u-L) M}^{u M-1}\left[p_{w-j}+n_{j}(w)\right] x_{j}(m) V_{r}\right]=\frac{1}{M} \sum_{j=(u-L) M}^{u M-1} \lambda_{j},
$$

where the last equation comes from the fact that each type is activated with equal probability for all activation patterns. Substituting $\Lambda_{c}$ into (7), we have

$$
\Lambda_{m}^{v}(u)=\lambda_{u}^{v} x_{u}(m, v)+\Lambda_{I E I}+\Lambda_{c} .
$$

Furthermore, based on (9) and (11), the decision criterion of the CML detector can be derived following the same approach as that of the GML detector.

\section{LML Detector}

The CML detector is implemented by exploiting statistical knowledge, its implementation, however, still imposes a high computational complexity on the MC system with nanomachine transceivers. For practical implementation, we propose an LML detector using a Viterbi-like algorithm.

Since the IEI coming from the current symbol has a slight impact on the detection, the effect of $\Lambda_{I E I}$ is ignored and (11) can be approximated as

$$
\Lambda_{m}^{v}(u)=\lambda_{u}^{v} x_{u}(m, v)+\Lambda_{c}
$$

Based on (9) and (12), an LML detector can be designed as

$$
\hat{\mathbf{X}}_{u}=\arg \max _{\widetilde{\mathbf{X}}_{u} \in \mathbf{S}} \sum_{m=1}^{M} \sum_{v=0}^{M-1} z_{m}^{v} \ln \left(\lambda_{u}^{v} \widetilde{x}_{u}(m, v)+\Lambda_{c}\right)
$$

$$
=\arg \underset{\widetilde{\mathbf{X}}_{u} \in \mathbf{S}}{\max } \sum_{m=1}^{M} \sum_{v=0}^{M-1} z_{m}^{v} \widetilde{x}_{u}(m, v) .
$$

According to (13), the LML detector selects the output symbol only dependent on the observations of the numbers of molecules, which reduces the computational complexity.

Apparently, the computational complexity of (13) is $\mathcal{O}(M !)$, increasing factorially with $M$, which is still intolerable as $M$ increases to a large value. For further reducing the computational complexity, we exploit a Viterbi-like algorithm [15] to reduce the search region. First, we initiate the trellis with $M+1$ stages and $M$ state transitions for accumulating the $M$ path metrics. At the $\beta$-th stage, there are $C(M, \beta)$ states labeled by the $\beta$-combinations of $M$ types, where $C(\cdot, \cdot)$ denotes the binomial coefficient. There exists a path if the state at the $(\beta-1)$-th stage is a subset of that at the $\beta$-th stage. Each path is associated with a path metric $T_{\beta, \alpha}$, where $\alpha$ is the complementary type of those two states. Then, when more than one path merge at the same state, we compare the accumulated metrics of all paths and keep the one with the maximum value only for further consideration. Finally, the $\alpha$ of each state transition on the surviving path represents the activated type. Hence, its computational complexity is $\mathcal{O}\left(M 2^{M-1}\right)$, which is less than that of the brute-force search in (13).

\section{PERformance AnAlysis}

In this section, we derive the upper bounds for the BER of the GML and CML detectors.

Assume $\mathbf{X}_{u}=\mathbf{S}_{k}$ is the transmitted pattern. According to (9), the detector yields an incorrectly detected symbol $\mathbf{S}_{l}$, when

$$
\begin{gathered}
\sum_{(m, v) \in \Omega_{k} \cup \Omega_{l}} z_{m}^{v} \ln \left(\Lambda_{m}^{v}\left(u \mid s_{k}(m, v)\right)\right) \leq \\
\sum_{(m, v) \in \Omega_{k} \cup \Omega_{l}} z_{m}^{v} \ln \left(\Lambda_{m}^{v}\left(u \mid s_{l}(m, v)\right)\right), \quad \forall l \neq k,
\end{gathered}
$$

where $\Omega_{k}=\left\{(m, v) \mid \forall m, \forall v, s_{k}(m, v)=1\right\}$. Therefore, the terms $(m, v) \in \Omega_{k} \cup \Omega_{l}$ represent that they are activated in $\mathbf{S}_{k}$ or $\mathbf{S}_{l}$. For $\mathbf{X}_{u}$, whether the $m$-th type at the $v$-th observation is activated or not leads to a different formulation of $\Lambda_{m}^{v}(u)$ :

$\Lambda_{m}^{v}(u)=\left\{\begin{array}{l}\lambda_{w}+i \Lambda_{I S I}+(1-i) \Lambda_{c}=\Lambda_{1}, x_{u}(m, v)=1, \\ \Lambda_{I E I}+i \Lambda_{I S I}+(1-i) \Lambda_{c}=\Lambda_{0}, x_{u}(m, v)=0,\end{array}\right.$

where $i=1$ or 0 gives the Poisson parameters of the GML detector or the CML detector. Hence, we can simplify (14) to

$$
\sum_{(m, v) \in \Omega_{k}-\Omega_{l}} z_{m}^{v} \ln \left(\frac{\Lambda_{1}}{\Lambda_{0}}\right)-\sum_{(m, v) \in \Omega_{l}-\Omega_{k}} z_{m}^{v} \ln \left(\frac{\Lambda_{1}}{\Lambda_{0}}\right) \leq 0,
$$

where the terms $(m, v) \in \Omega_{k}-\Omega_{l}$ represent that they are activated in $\mathbf{S}_{k}$ but inactivated in $\mathbf{S}_{l}$. When $\Lambda_{m}^{v}(u) \gg 20$, the Gaussian approximation of the Poisson distribution can be used, i.e., $z_{m}^{v} \sim \mathcal{N}\left(\Lambda_{m}^{v}(u), \Lambda_{m}^{v}(u)\right)$. Let the Gaussian distributed random variable $Y$ denote the left-hand side of (15), whose mean and variance are given by

$$
\begin{aligned}
& \mu_{Y}=\sum_{(m, v) \in \Omega_{k}-\Omega_{l}} \Lambda_{m}^{v} \ln \left(\frac{\Lambda_{1}}{\Lambda_{0}}\right)-\sum_{(m, v) \in \Omega_{l}-\Omega_{k}} \Lambda_{m}^{v} \ln \left(\frac{\Lambda_{1}}{\Lambda_{0}}\right), \\
& \sigma_{Y}^{2}=\sum_{(m, v) \in \Omega_{k}-\Omega_{l}} \Lambda_{m}^{v} \ln ^{2}\left(\frac{\Lambda_{1}}{\Lambda_{0}}\right)+\sum_{(m, v) \in \Omega_{l}-\Omega_{k}} \Lambda_{m}^{v} \ln ^{2}\left(\frac{\Lambda_{1}}{\Lambda_{0}}\right) .
\end{aligned}
$$


TABLE I

SYSTEM PARAMETERS

\begin{tabular}{lcc}
\hline Parameter & Variable & Value \\
\hline Radius of receiver & $r$ & $1 \mu \mathrm{m}$ \\
Tx-Rx Distance & $d$ & $20 \mu \mathrm{m}$ \\
Diffusion coefficient & $D$ & $2.2 \times 10^{-9} \mathrm{~m}^{2} / \mathrm{s}$ \\
Symbol duration & $T_{s}$ & {$[0.025,0.75] \mathrm{s}$} \\
Emitted molecules per bit & $E_{s} / b$ & {$[0.4,16] \times 10^{5}$} \\
Types of molecules & $M$ & $\{1,2,4,8\}$ \\
\hline
\end{tabular}

Based on (16), the conditional pairwise error probability (PEP) can be expressed as

$$
\operatorname{Pr}\left(\mathbf{S}_{k} \rightarrow \mathbf{S}_{l} \mid \mathbf{X}_{u-L}, \ldots, \mathbf{X}_{u-1}\right)=Q\left(\mu_{Y} / \sigma_{Y}^{2}\right),
$$

where $Q(x)=(2 \pi)^{-1 / 2} \int_{x}^{\infty} e^{-t^{2} / 2} d t$. Assume that all symbols are activated with equal probability. The unconditioned PEP can then be readily derived from (17) as

$$
\operatorname{Pr}\left(\mathbf{S}_{k} \rightarrow \mathbf{S}_{l}\right)=\frac{1}{N^{L}} \sum_{X_{u-L}, \ldots, X_{u-1} \in \mathbf{S}^{L}} Q\left(\mu_{Y} / \sigma_{Y}^{2}\right) .
$$

Finally, according to the union-bounding technique, the BER can be upper bounded by

$$
P_{e} \leq \frac{1}{b N} \sum_{k=1}^{N} \sum_{l=1, l \neq k}^{N} \operatorname{Pr}\left(\mathbf{S}_{k} \rightarrow \mathbf{S}_{l}\right) e\left(\mathbf{S}_{k} \rightarrow \mathbf{S}_{l}\right),
$$

where $e\left(\mathbf{S}_{k} \rightarrow \mathbf{S}_{l}\right)$ denotes the number of error bits corresponding to the pairwise error event.

\section{Numerical Results}

In this section, we present the BER performance of the proposed MTPSK schemes with various detectors over the diffusion channels. We choose the binary (B-)CSK, MoSK, DMoSK, and 4-PPM schemes as the benchmarks, where both BCSK and D-MoSK schemes employ the optimal threshold that minimizes the BER, MoSK employs the LML detector, and 4-PPM employs 4 different positions to determine the intended symbol [5]. For fairness, the BER performance is evaluated versus the number of emitted molecules per bit $E_{s} / b$, where $E_{s}$ and $b$ denote the numbers of emitted molecules and transmitted bits per symbol, respectively. Note that BCSK and D-MoSK transmit bit- 1 by emitting $2 E_{s} / b$ molecules, since it transmits bit- 0 by emitting no molecules and the probability of transmitting bit- 1 is assumed to be 0.5 . The simulation parameters of the MC system are given in Table I, where $E_{s}=M E_{e}$ for MTPSK. Moreover, based on [12], the length $L_{e}$ of interference from previous emissions is restricted to

$$
L_{e}=\arg \min _{w-j}\left\{p_{w-j} / p_{0} \leq 0.4 \%\right\} .
$$

Fig. 1 compares the analytical BER results with their simulated counterparts of the CML and GML detectors, and depicts the BER performance of MTPSK with three various detectors considering $M=2$ or 4 at the same data rate. It can be observed that when $M=2$ the analytical curves match well with the simulation counterparts. When $M=4$ the derived upper bounds are asymptotically tight as more molecules are emitted. The BER gaps can be understood by the fact that

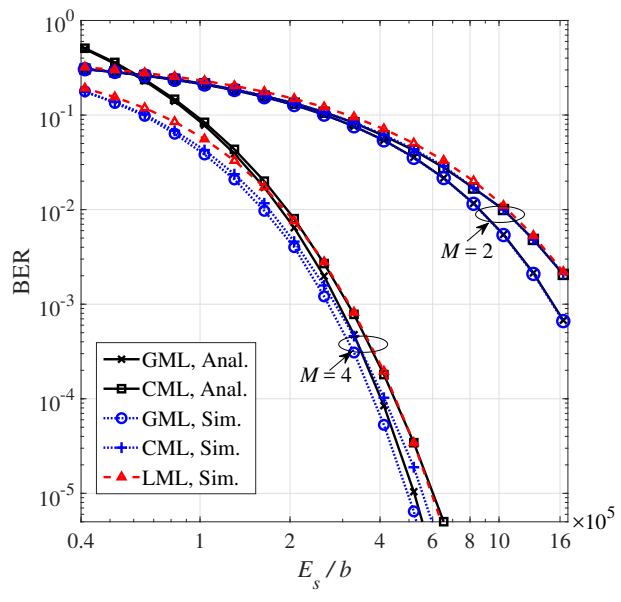

Fig. 1. Performance of the MTPSK schemes with various detectors and the validation of analytical results of the CML and GML detectors, when $T_{s}=$ $0.4 \mathrm{~s}$ or $0.1 \mathrm{~s}$ corresponding to $M=4$ or 2 .

the union-bounding technique considers overlapping decision regions. Additionally, the LML detector achieves similar BER performance to the GML and CML detectors. The reason behind is that the IEI ignored by the LML detector cannot accumulate, which has a negligible effect on detection. These simulation results demonstrate that the MTPSK employing LML detector mitigates ISI effectively while maintaining low computational complexity.

Fig. 2 shows the BER performance of MTPSK employing the LML detector when different data rates, denoted by $c$ in the figures, are considered, where the BCSK, MoSK, D-MoSK, and 4-PPM schemes are regarded as benchmarks. For a fair comparison, MTPSK, MoSK, D-MoSK, and 4-PPM all employ $M=4$ types of molecules, where 4-PPM use different types of molecules as orthogonal channels, and hence, $b=[4,2,4,8]$, respectively. With $b=1$ for BCSK, $T_{s}$ is adjusted to guarantee all considered schemes with the same data rate. According to (20), we set the ISI length $L=L_{e} / M$

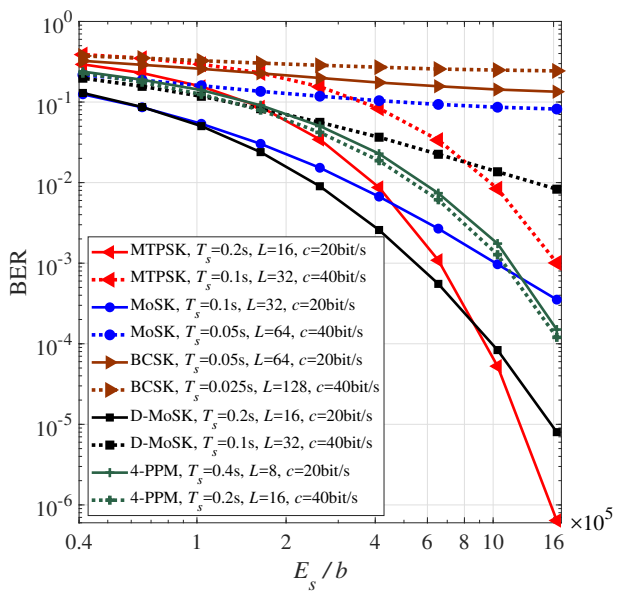

Fig. 2. BER comparison among MoSK, BCSK, D-MoSK, 4-PPM, and MTPSK, when $c=20 \mathrm{bit} / \mathrm{s}$ or $40 \mathrm{bit} / \mathrm{s}$ and $M=4$ are considered.

for MTPSK since there are $M$ emissions within each symbol duration, and $L=L_{e}$ for the other schemes. As shown in Fig. 2, the BER performance of MTPSK outperforms that 
of D-MoSK at high $E_{s} / b$. This can be explained by two reasons: for D-MoSK, its larger $T_{s}$ than that of BCSK and MoSK reduces the ISI; for MTPSK, it is more accurate to detect molecular numbers of $M$ instants simultaneously than to detect multiple BCSK streams individually in D-MoSK. Moreover, the intersection points of BER curves of MTPSK and MoSK appear at medium $E_{s} / b$ values. The reason behind the above superiority is that the molecular types within a symbol are non-repeated for MTPSK, which further enlarges the transmission interval of molecules of the same type statistically in comparison with MoSK. At low $E_{s} / b$, the BER performance of MTPSK underperforms that of MoSK and D-MoSK. This is because although MTPSK is capable of mitigating ISI effectively, the dominant interference at low $E_{s} / b$ is counting noise, not ISI. Additionally, the BER performance of MTPSK is always much better than that of BCSK. This is because that the performance of thresholdbased detection in BCSK is sensitive to the ISI. It is worth noting that if we compare MTPSK with higher order formats of CSK, such as quadrature (Q-)CSK, the BER performance gap will be larger. Since when the average number of emitted molecules per transmission is normalized, for QCSK the distances between adjacent thresholds decrease compared with BCSK, which increases the possibility of erroneous detection. Finally, MTPSK underperforms 4-PPM when data rate equals 40bit/s; however, its superiority emerges at a lower data rate. This is because, unlike other modulation schemes, an increase of data rate (i.e., a decrease of symbol duration) for 4-PPM may cause a beneficial effect on the BER performance.

Fig. 3 compares the BER performance of MTPSK employing the LML detector with that of MoSK and D-MoSK, when different numbers of molecular types are considered. It can be seen that when $M=8$ the intersection points of BER curves of MTPSK and the other two schemes appear at a smaller $E_{s} / b$ value compared with those when $M=4$, which demonstrates that the gap of BER performance will increase as more number of types are available in the system. Moreover, the BER performance of MoSK surpasses that of D-MoSK when $M=8$, since the increased $M$ further reduces the possibility that molecules of the same type accumulate in a short time.

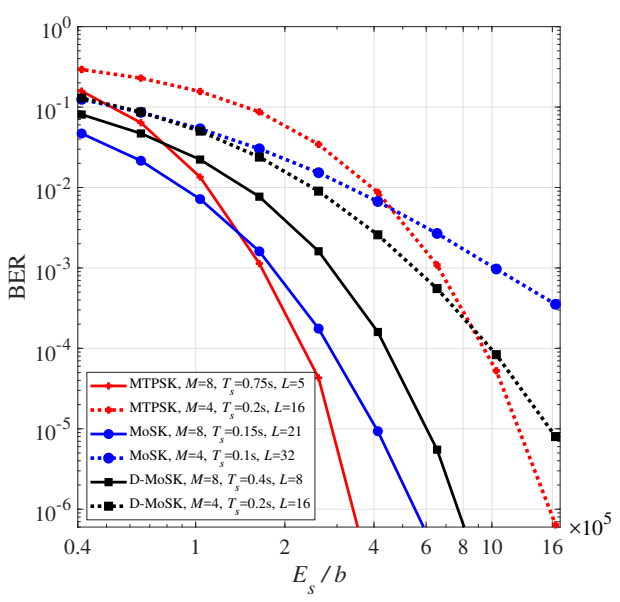

Fig. 3. BER comparison among MoSK, D-MoSK, and MTPSK, when $M=8$ or 4 and $c=20 \mathrm{bit} / \mathrm{s}$ are considered.

\section{CONCLUSION}

In this paper, we have proposed the MTPSK scheme, which encodes information on the permutations of multiple types of molecules. Three detectors have been proposed for MTPSK to show its potential performance, and design of high-efficiency low-complexity detector. The analytical error upper bounds are calculated and BER results are obtained by Monte Carlo simulations.

The MTPSK scheme in the space domain will be considered as our future work.

\section{REFERENCES}

[1] N. Farsad et al., "A comprehensive survey of recent advancements in molecular communication," IEEE Commun. Surveys Tut., vol. 18, no. 3, pp. 1887-1919, 3rd Quart. 2016.

[2] B. Tepekule, A. E. Pusane, H. B. Yilmaz, C.-B. Chae, and T. Tugcu, "ISI mitigation techniques in molecular communication," IEEE Trans. Mol. Biol. Multi-Scale Commun., vol. 1, no. 2, pp. 202-216, June 2015.

[3] R. Mosayebi et al., "Type-based sign modulation and its application for ISI mitigation in molecular communication," IEEE Trans. Commun., vol. 66, no. 1, pp. 180-193, Jan. 2018.

[4] M. S. Kuran, H. B. Yilmaz, T. Tugcu, and I. F. Akyildiz, "Modulation techniques for communication via diffusion in nanonetworks," in Proc. IEEE ICC, Kyoto, Japan, June 2011, pp. 1-5.

[5] B. C. Akdeniz, A. E. Pusane, and T. Tugcu, "Position-based modulation in molecular communications," Nano Commun. Netw., vol. 16, pp. 60-68, June 2018.

[6] Y. Huang, M. Wen, L.-L. Yang, C.-B. Chae, and F. Ji, "Spatial modulation for molecular communication," IEEE Trans. NanoBiosci., vol. 18, no. 3, pp. 381-395, July 2019.

[7] M. C. Gursoy, E. Basar, A. E. Pusane, and T. Tugcu, "Index modulation for molecular communication via diffusion systems," IEEE Trans. Commun., vol. 67, no. 5, pp. 3337-3350, May 2019.

[8] M. H. Kabir, S. M. R. Islam, and K. S. Kwak, "D-MoSK modulation in molecular communications," IEEE Trans. Nanobiosci., vol. 14, no. 6 , pp. 680-683, Sept. 2015.

[9] B. Atakan, S. Galmes, and O. B. Akan, "Nanoscale communication with molecular arrays in nanonetworks," IEEE Trans. NanoBiosci., vol. 11, no. 2, pp. 149-160, June 2012.

[10] N.-R. Kim et al., "Novel modulation techniques using isomers as messenger molecules for nano communication networks via diffusion," IEEE J. Sel. Areas Commun., vol. 31, no. 12, pp. 847-856, Dec. 2013.

[11] M. Turan et al., "Channel model of molecular communication via diffusion in a vessel-like environment considering a partially covering receiver," in Proc. IEEE Int. Black Sea Conf. Commun. Netw. (BlackSeaCom), Batumi, Georgia, June 2018, pp. 1-5.

[12] L. Shi and L.-L. Yang, "Error performance analysis of diffusive molecular communication systems with on-off keying modulation," IEEE Trans. Mol., Biol. Multi-Scale Commun., vol. 3, no. 4, pp. 224-238, Dec. 2017.

[13] I. Llatser, A. Cabellos-Aparicio, M. Pierobon, and E. Alarcon, "Detection techniques for diffusion-based molecular communication," IEEE J. Sel. Areas Commun., vol. 31, no. 12, pp. 726-734, Dec. 2013.

[14] M. Pierobon and I. F. Akyildiz, "Diffusion-based noise analysis for molecular communication in nanonetworks," IEEE Trans. Signal Process., vol. 59, no. 6, pp. 2532-2547, June 2011.

[15] M. Wen, E. Başar, Q. Li, B. Zheng, and M. Zhang, "Multiple-mode orthogonal frequency division multiplexing with index modulation," IEEE Trans. Commun., vol. 65, no. 9, pp. 3892-3906, Sept. 2017. 